\title{
POEMS syndrome
}

INSERM

\section{Source}

INSERM. (1999). Orphanet: an online rare disease and orphan drug data base. POEMS syndrome. ORPHA:2905

POEMS syndrome is a paraneoplastic syndrome characterized by polyradiculoneuropathy $(P)$, org anomeg aly $(O)$, endocrinopathy $(E)$, clonal plasma cell disorder $(M)$, and skin changes (S). Other features include papilledema, extravascular volume overload, sclerotic bone lesions, thrombocytosis/erythrocytosis, and elevated VEGF levels. 